\title{
Mathematical Modeling of Solar Energy Fresh Air System
}

\author{
Lili Wang ${ }^{1,2}$ a $^{*}$, Naixiu Ding ${ }^{1, b}$ and Meijia Wang ${ }^{3, c}$ \\ ${ }^{1}$ Qingdao University of Science and Technology, Qingdao, China \\ ${ }^{2}$ Qingdao Hanhe Cable Co., Ltd. Qingdao,China \\ ${ }^{3}$ Qingdao Publishing \& Media Co., Ltd., Qingdao, China \\ awllqust@126.com, ㅁnxding1717@163.com, cw.mj@163.com
}

Keywords: Solar energy; Fresh air; LHTS; Mathematical model

\begin{abstract}
Solar energy fresh air system is a complex thermal system which is mainly composed of three parts: heat collecting system, LHTS (latent heat thermal storage) device storage / exothermic system, fresh air system. As it is affected by multiple factors such as sun light intensity, air state parameters, air velocity, water temperature and flow rate, it is extremely difficult to establish exact mathematical model and obtain accurate system parameters. Therefore, in this paper, on the reasonable assumption, we establish the mathematical model including LHTS device, fan coil, according to Energy Conversion Method (ECM), computational fluid dynamics (CFD), the Numerical Heat Transfer (NHT) and phase change energy storage theory.
\end{abstract}

\section{Introduction}

As shown in Fig.1,solar energy fresh air system is mainly composed of three parts: heat collection system, heat storage system, fresh air heating system, and each part has its own characteristics[1-2], of which the process model can be realized generally by two methods, one is to establish the differential equations controlled through the analysis of the controlled process, using some known laws, theorems and principle, and then obtained the transfer function model; the other is called system identification method, which makes use of input output experimental data to establish the transfer function model[3] .

In this paper, we firstly establish the differential equation of various part of the fresh air system based on the law of energy conservation, and then transform it into transfer function and determine the model order .

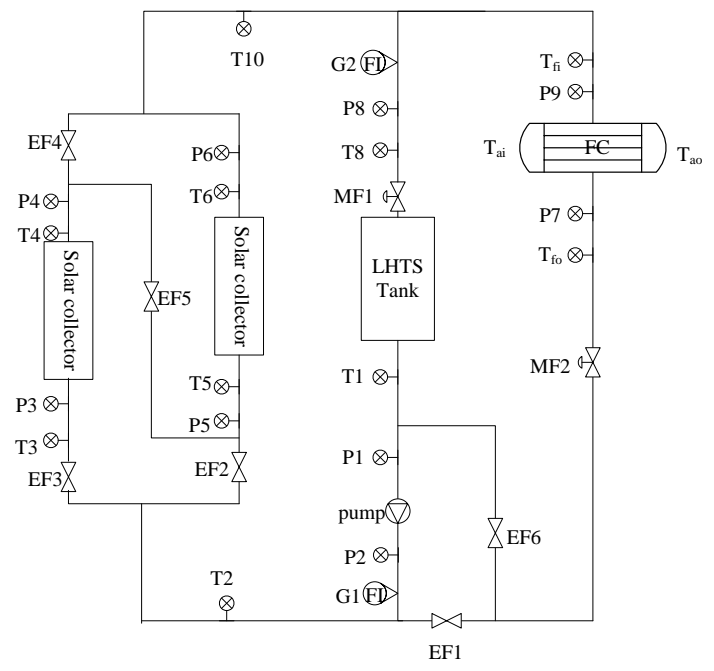

Figure 1. Solar energy fresh air system with solar collectors and LHTS tank 


\section{Mathematical Model of Phase Change Heat Storage Device}

Phase Change Heat Storage Material Computational Model. The phase change heat storage device mainly comprises a plate shaped heat storage body and water, and the heat exchange mode is a heat exchange between the plate and water. Therefore, both of the mathematical models should be respectively established.

The LHTS device is shown in Fig. 2

Governing Equation. The hot water (heating medium water) flows along $\mathrm{X}$ direction, and makes direct heat transfer with form-stable phase change material filled in the heat storage device. In order to facilitate the establishment and derivation of the mathematical model, we firstly make the following assumptions:

(1) The length of storage materials is far longer than the thickness; the internal temperature distribution of heat storage materials only depends on thickness and height, and independent of the length;

(2) Due to the small size of the encapsulated phase change material, assume that the internal heat transfer is controlled by heat conduction; the internal heat transfer is two-dimensional heat conduction problems, ignoring the convective heat transfer inside the material storage;

(3) ignore the volume change of regenerative material transformation with equal density;

(4) The properties change of the phase change heat storage material is not considered, such as the decline of performance.

(5) It is assumed that the material in the phase change thermal storage device is homogeneous and isotropic;

(6) It is assumed that the melting point of the heat storage material is certain, that is, the isothermal transformation;

The heat transfer process of phase change heat storage device is actually a complex three-dimensional phase change problem. The heat storage device is side by side structure, as shown in in Fig .2, in this paper we take one thermal storage unit to study(the length of L, thickness of D/2) as shown in Fig. 3.[4].

Concerning with the geometric symmetry of the heat storage body and both sides of the heat medium water, the coordinate system is established in the plate heat storage plate center line, and along the centerline of the board is consiered as adiabatic; In regenerator region, it is dealt with two-dimensional heat conduction and heat conduction along plate storage heater length $\mathrm{Z}$ direction is ignored.

Under above assumptions, the differential equation of the heat storage material can be given as follows:

\section{Heat Storage Area:}

$$
\rho_{P} c_{P} \frac{\partial T_{P}}{\partial t}=\frac{\partial}{\partial x}\left(\lambda_{P} \frac{\partial T_{P}}{\partial x}\right)+\frac{\partial}{\partial y}\left(\lambda_{P} \frac{\partial T_{P}}{\partial y}\right)
$$




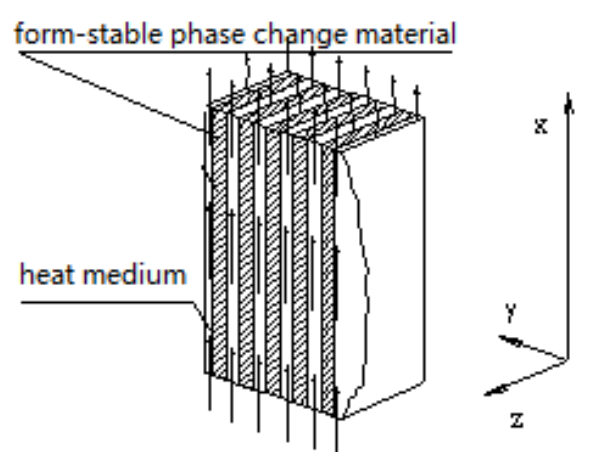

Figure 2. LHTS device side by side structure

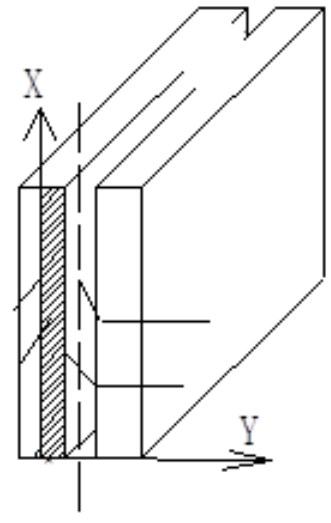

Figure 3. Single heat storage unit coordinate system

\section{Boundary Condition.}

The lower surface of the plate $(y=0)$ temperature is the same as the inlet water temperature: [1].

$$
\left.T_{p}\right|_{y=0}=T_{i n}
$$

The plate center surface $(\mathrm{x}=0)$ is considered as adiabatic.

$$
\left.\frac{\partial T}{\partial x}\right|_{x=0}=0
$$

On the right side surface $(x=D / 2)$ of the plate is considered as a convective heat transfer

$$
-\left.\lambda_{p} \frac{\partial T_{P}}{\partial y}\right|_{x=D / 2}=h\left(\left.T_{p}\right|_{x=D / 2}-T_{f}\right)
$$

The upper end surface of the plate $(\mathrm{y}=\mathrm{L})$ is considered as a convective heat transfer:

$$
-\left.\lambda_{p} \frac{\partial T_{P}}{\partial y}\right|_{y=L}=h\left(\left.T_{p}\right|_{y=L}-T_{f}\right)
$$

Initial temperature of phase change heat storage plate:

$$
\left.T_{P}\right|_{t=0}=T_{0}
$$

Calculation Model of Heat Medium Water in Phase Change Thermal Storage Device. As mentioned before, the water is the heat transfer medium of the phase change heat storage device, and the hot water flows along the $\mathrm{X}$ direction.

Control Equation. In order to facilitate the establishment and derivation of the mathematical model, we firstly make the following assumptions:

(1) Convective heat transfer coefficient between the heat storage material and the hot water is constant;

(2) Only consider the temperature variation along the flow direction of $\mathrm{X}$.

Under the above assumptions, the differential equations can be given as follows:

Heat medium water: 
$\rho_{f} c_{f}(1-\varepsilon)\left(\frac{\partial T_{f}}{\partial t}+u \frac{\partial T_{f}}{\partial x}\right)=h A\left(\left.T_{p}\right|_{x=D / 2}-T_{f}\right)$

Boundary Condition.

The hot water inlet temperature and flow rate changes with time:

$\left.T_{f}\right|_{y=0}=T_{i n}(t), u=u(t)$

The initial temperature of heat fluids in the LHTS device

$\left.T_{f}\right|_{t=0}=T_{0}$

\section{Mathematical Model of Fan Coil}

Fan Coil Dynamic Differential Equation. The Fan coil is the end heat sink of the solar energy fresh air system, which makes heat transfer between the fresh air and the heated fluid. Fan coil heat transfer is extremely complicated, of which the hot and cold fluid respectively flows in and outside the coil, and the heat passes through the heated fluid to the tube wall in the form of convection and heat radiation. After the tube wall being heated, it also makes heat transfer with the cold fluid in form of convection and heat radiation, which achieve heat transfer inside the hot and cold water and fresh air outside. In the case that some parameters are consistent such as the structure, the heat exchange area and the mass flux, the heat exchange rate of the fan coil unit is mainly related to the inlet temperature of hot water and the inlet temperature of fresh air. [5]

The schematic diagram of fan coil principle is shown in Fig. 4

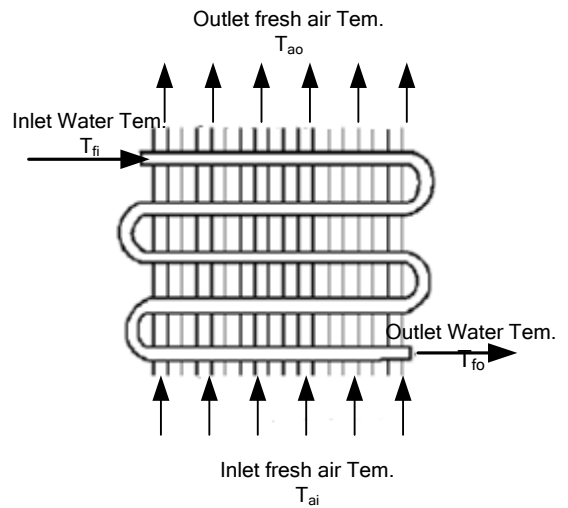

Figure 4. Schematic diagram of fan coil heat exchange principle

In order to establish the mathematical model of the fan coil, some necessary assumptions are as follows:

(1) Dry air and water vapor in the air is considered as an ideal gas, and ignore the fluid density and specific heat capacity changes;

(2) Air flow evenly transfers through the coil heat exchanger, and the heat transfer coefficient between air and heat exchangers outer surface is constant, which only varies with the flow velocity of fluid;

(3) Fan coil inlet and outlet air flow are the same;

(4) Ignore the mutual influence between two vertical adjacent pipes of fan coil unit, and the fluid temperature inside the tube only changes along the length direction, the temperature distribution along the radius direction is uniform. 
Consequently, according to the heat balance principle, in which the system's energy intake is equal to the system's output energy, the dynamic relationship between the inlet and outlet parameters of the fan coil heat exchanger can be expressed as:

$$
c_{f c} \frac{d T_{a o}}{d t}=\partial_{a}\left(T_{a i}-T_{a o}\right)+\rho_{f} c_{f} w_{f}\left(T_{f i}-T_{f o}\right)+q
$$

\section{Fan Coil Control Model}

As we all know, the system can be decomposed into the steady state and the transient state, that is, every state can be represented by the steady and the transient value of the system $\left(\overline{T_{a o}}\right.$ and $\overline{w_{f}}$ are the steady value).

$$
\left\{\begin{array}{l}
w_{f}=\overline{w_{f}}+\Delta w_{f} \\
T_{a o}=\overline{T_{a o}}+\Delta T_{a o}
\end{array}\right.
$$

Then Eq.10 can be rewritten as follows:

$$
c_{f c} \frac{d \Delta T_{a o}}{d t}=\partial_{a}\left(T_{a i}-\overline{T_{a o}}-\Delta T_{a o}\right)+\rho_{f} c_{f}\left(\overline{w_{f}}+\Delta w_{f}\right)\left(T_{f i}-T_{f o}\right)+q
$$

When the system is in stable condition:

$$
0=\partial_{a}\left(T_{a i}-\overline{T_{a o}}\right)+\rho_{f} c_{f} \overline{w_{f}}\left(T_{f i}-T_{f o}\right)+q
$$

Taking Eq.13into Eq.12, we can obtain:

$$
c_{f c} \frac{d \Delta T_{a o}}{d t}=-\partial_{a} \Delta T_{a o}+\rho_{f} c_{f} \Delta w_{f}\left(T_{f i}-T_{f o}\right)
$$

Get the Laplace transform of formula Eq.14

$$
c_{f c} s \Delta T_{a o}(s)=-\partial_{a} \Delta T_{a o}(s)+\rho_{f} c_{f} \Delta w_{f}(s)\left(T_{f i}-T_{f o}\right)
$$

After the rearrangement, we obtain:

$$
\frac{\Delta T_{a o}(s)}{\Delta w_{f}(s)}=\frac{\rho_{f} c_{f}\left(T_{f i}-T_{f o}\right)}{c_{f c} s+\partial_{a}}
$$

Therefore, the data model of the fan coil unit can be estimated accurately by a first-order plus time delay model:

$$
\frac{T_{a o}(s)}{w_{f}(s)}=\frac{k}{T s+1} e^{-\tau s}
$$

\section{Summary}

In this paper, we studied a kind of fresh air system with solar collectors and LHTS device. According to the system operation characteristics we have investigated the mathematical models of LHTS device and fan coil are finally established. 


\section{Acknowledgements}

In this paper, the research was supported by the Natural Science Foundation of Shandong Province, China (ZR2015PF008)

\section{References}

[1] Z.Y LI, Theoretical and experimental study on solar heating system with phase change thermal storage (Ph.D, Beijing University of Technology, 2011. ( (In Chinese)),P34

[2] L.L.Wang, N.X.Ding and M.J.WANG, Proc. International Conference on Intelligent Systems Research and Mechatronics Engineering (Zhengzhou,china, April11-13,2015).p963

[3] CZ. Fang, DY.Xiao: Process Identification (Tsinghua University press1988),p84 (In Chinese)

[4] Vikram.D, Kaushik. S, Prashanth. V, Nallusamy.N. An improvement in the solar water heating systems by thermal storage using phase change materials. International Solar Energy Conference, Proceedings of the ASME International Solar Energy Conference, ISEC2006, 2007, 409-416.

[5] Wang lili, control system study on solar heating system with phase change thermal storage (Ph.D. Beijing university of posts and communications, 2010. )(In Chinese) 